Annales Geophysicae (2002) 20: 1487-1498 C European Geophysical Society 2002

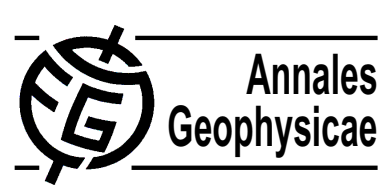

\title{
Morning sector drift-bounce resonance driven ULF waves observed in artificially-induced HF radar backscatter
}

\author{
L. J. Baddeley ${ }^{1}$, T. K. Yeoman ${ }^{1}$, D. M. Wright ${ }^{1}$, J. A. Davies ${ }^{1}$, K. J. Trattner ${ }^{2}$, and J. L. Roeder ${ }^{3}$ \\ ${ }^{1}$ Department of Physics and Astronomy, University of Leicester, University Road, Leicester LE1 7RH, U K \\ ${ }^{2}$ Lockheed Martin ATC, Palo Alto, USA \\ ${ }^{3}$ The Aerospace Corporation, Los Angeles, CA, USA
}

Received: 24 October 2001 - Revised: 5 March 2002 - Accepted: 22 March 2002

\begin{abstract}
HF radar backscatter, which has been artificiallyinduced by a high power RF facility such as the EISCAT heater at Troms $\varnothing$, has provided coherent radar ionospheric electric field data of unprecedented temporal resolution and accuracy. Here such data are used to investigate ULF wave processes observed by both the CUTLASS HF radars and the EISCAT UHF radar. Data from the SP-UK-OUCH experiment have revealed small-scale (high azimuthal wave number, $m \approx-45$ ) waves, predominantly in the morning sector, thought to be brought about by the drift-bounce resonance processes. Conjugate observations from the Polar CAMMICE instrument indicate the presence of a non-Maxwellian ion distribution function. Further statistical analysis has been undertaken, using the Polar TIMAS instrument, to reveal the prevalence and magnitude of the non-Maxwellian energetic particle populations thought to be responsible for generating these wave types.
\end{abstract}

Key words. Ionosphere (active experiments; wave-particle interactions) Magnetospheric physics (MHD waves and instabilities)

\section{Introduction}

Ultra Low Frequency (ULF) waves can be classified into two basic types with respect to their energy generation mechanisms. The first type is characterized by the small magnitude of its effective azimuthal wave numbers $m$, (or equivalently a large scale size in the azimuthal direction) and has an energy source external to the Earth's magnetosphere. This can include such mechanisms as the Kelvin-Helmholtz instability on the magnetopause, solar wind buffeting or an impulsive increase in solar wind pressure at the front of the magnetopause. The second type of waves is conversely characterized by its high magnitude $m$ numbers. The generation mech-

Correspondence to: L. J. Baddeley

(Lisa.Baddeley@ion.le.ac.uk) anism, thought to be responsible for these waves, is internal to the magnetosphere in the form of wave-particle interactions with non-Maxwellian particle distributions. These interactions lead to perturbations in the electric and magnetic fields in the magnetosphere and ionosphere when free energy is available to the wave. It has been suggested (e.g. Southwood, 1976; Hughes et al., 1978) that the part of the ion distribution function (IDF) which is able to feed energy into the wave is that where the IDF has a positive gradient, i.e. $\partial f / \partial W>0$, where $f$ represents the particle distribution function and $W$ the particle energy. Such non-Maxwellian or "bump-on-the-tail" IDFs can be created quite often by naturally occurring processes in the magnetosphere, such as substorm-associated particle injections. Subsequent to such an injection, the ions will gradient-curvature drift westwards, thus constituting part of the global ring current where they will on occasion match the local drift-bounce resonance condition (Southwood et al., 1969),

$\omega_{\text {wave }}-m_{\text {wave }} \omega_{\text {drift }}=N \omega_{\text {bounce }}$

where $N$ is an integer (usually \pm 1 ) and $\omega_{\text {wave }}, \omega_{\text {bounce }}$, and $\omega_{\text {drift }}$ are the angular frequencies of the wave, the proton bounce and the proton azimuthal drift, respectively. Such wave-particle interactions are a process of fundamental importance in collisionless astrophysical plasmas. The ionosphere modifies the magnetospheric ULF wave signature, leading to both rotation and attenuation of the magnetic signature of the wave as detected on the ground (e.g. Hughes and Southwood, 1976; Hughes, 1983). This attenuation of the pulsation magnetic perturbation below the ionosphere is proportional to $e^{-k z}$ (e.g. Hughes and Southwood, 1976) where $k$ is the field perpendicular component of the wave number and $z$ is the E-region height. The result of this modification is that ground magnetometer data is far from optimal for the study of high- $m$ ULF waves and that direct observation in the ionosphere or magnetosphere is usually required. 


\section{Previous observations}

There have been several studies of high $m$ wave phenomena in both the post-noon and pre-noon sectors. It is observed that the characteristics of morning and afternoon sector waves are very different. While the underlying processes and driving mechanisms that occur in the afternoon sector are relatively well understood and agreed on, much controversy still surrounds morning sector wave phenomena.

In the afternoon sector fundamental wave modes have been identified as interacting with particle populations through wave particle interactions. Examples of such a fundamental mode are the storm time Pc5 pulsations that have been seen in STARE (the Scandinavian Twin Auroral Radar Experiment; Greenwald et al., 1978) data (Allan et al., 1982, 1983); these are compressional waves, of high $m$ number $(m=-20$ to -80$)$ and frequency in the Pc5 range $(1.7-6.7 \mathrm{mHz})$, which are associated with a drift resonance source mechanism. Drift resonance involves fundamental wave modes with the electric field symmetric about the equator (i.e. an equatorial electric field antinode). Stormtime Pc5s are observed in the dusk sector during magnetically disturbed intervals. Similar waves, but with an equatorward phase motion, have been seen in SABRE (the Sweden And Britain auroral Radar Experiment; Nielsen et al., 1983) and BARS (Bistatic Auroral Radar System; McNamara et al., 1983) coherent radar systems in the dusk sector by Yeoman et al. (1992) and Grant et al. (1992), respectively.

Yeoman and Wright (2001) previously presented a study of three waves observed in artificially-induced HF radar backscatter that occurred in the post-noon sector, from the SP-UK-OUCH EISCAT campaign. Of the three waves observed, one was concluded to have a driving mechanism external to the Earth's Magnetosphere while the remaining two were demonstrated to have been brought about through wave-particle interactions. The mechanisms were identified as drift $(N=0)$ resonance and drift-bounce $(N=1)$ resonance, respectively, with evidence presented indicating how the wave evolved from the fundamental (drift mode) to the second harmonic (drift-bounce) mode. While they did not present any particle data they did surmise, using the drift bounce resonance equation, that a westward drifting particle population with a bump-on-the-tail located between 35 and $45 \mathrm{keV}$ could be responsible for driving the latter two waves.

In the morning sector, giant ' $P g$ ' pulsations form another class of particle driven ULF waves of interest, which have been observed on the ground as well as by orbiting satellites at times when geomagnetic conditions are quiet. $P g$ s are an example of a particle-driven ULF wave that may be studied with ground-based magnetometers due to their large amplitude. Chisham and Orr (1991) presented a statistical study of 34 of these events observed on the EISCAT (European Incoherent Scatter) magnetometer cross network in northern Scandinavia; they found a peak in occurrence of these waves in the dawn/pre-noon sector while no events were observed in the afternoon. The average value of the azimuthal wave number was about -26 for the 34 events. $P g$ s have been related to drift-bounce resonance mechanisms with both symmetric (e.g. Takahashi et al., 1992) and anti-symmetric (e.g. Chisham and Orr, 1991) wave modes, hence the waveparticle interaction responsible for their generation remains controversial.

Recent results from DOPE (the DOppler Pulsation Experiment; Wright et al., 1997) at Tromsø, northern Norway have demonstrated the existence of significant new populations of high- $m$ waves in both the morning and afternoon sectors (Yeoman et al., 2000). In this case the morning sector waves were the most populous and had $m$-numbers of order 100, high enough to screen the waves completely from ground magnetometers.

The structure and occurrence of particle-driven waves have been studied by a number of authors in the magnetosphere with in situ spacecraft data, for both case studies (e.g. Hughes et al., 1979; Takahashi et al., 1990) and statistically (e.g. Kokubun, 1985; Takahashi et al., 1985; Anderson et al., 1990; Woch et al.,1990; Engebretson et al., 1992; Lessard et al., 1999). These studies are largely consistent with the ground-based results, identifying the strongest populations of particle-driven waves in the dusk sector.

To date, there are very few publications which present both ground-based data and conjugate satellite data for such wave events. A recent paper by Wright et al. (2001) reported multi-instrument observations of a $P g$ pulsation. The wave occurred in the morning sector and was detected simultaneously on the ground by the International Monitor for Auroral Geomagnetic Effects (IMAGE) magnetometer network and in the ionosphere by DOPE. Shortly before the commencement of the $P g$, the particle instruments onboard the Polar spacecraft detected a non-Maxwellian or 'bump-on-the-tail' proton distribution drifting westward. In the paper they conclude that the $P g$ is likely to be the result of a drift-bounce resonance occurring between an even mode standing wave and energetic particles of around $7 \mathrm{keV}$, at the lower energy edge of the unstable distribution (where $\partial f / \partial W>0$ ).

Controversially, a recent paper by Glassmeier et al. (1999), see also (Mann and Chisham, 2000; Glassmeier, 2000) presented direct measurements of drifting non-Maxwellian particle populations made by the GOES 2 spacecraft and ground observations from the Scandanavian Magnetometer Array in the morning sector. In the paper they inferred that the resonance equation could be satisfied by a non-integer value of $N$ if the equation was modified slightly, thus, invoking an odd-mode fundamental field line oscillation interaction.

Recent theoretical models outlined in Ozeke and Mann (2001) predict an asymmetry between these properties of high $m$ waves in the morning and afternoon sectors. Their model provides excellent agreement with observations of stormtime Pc5 pulsations in the afternoon sector and $P g \mathrm{~s}$ in the morning sector, invoking $N=0$ and $N=1$ modes for the post-noon and pre-noon sector phenomena, respectively.

In this paper, data are analyzed from both ground based and satellite instrumentation in the form of data from the CUTLASS and EISCAT radars along with conjugate particle measurements made by the Polar spacecraft CAMMICE 
particle detector for a $P g$-like event in the morning sector. Statistical data are also presented from the TIMAS particle detector also onboard the Polar spacecraft, but with a slightly lower energy range than that of CAMMICE.

\section{Instrumentation}

\subsection{CUTLASS}

The radar data presented here result from the generation of artificial ionospheric HF coherent backscatter. It has been previously documented that the EISCAT Heating facility located at Troms $\varnothing$, Norway (Rietveld et al., 1993) is capable of generating artificial field-aligned irregularities using high power HF radio waves (e.g. Robinson, 1989) that are detectable by both coherent and incoherent scatter radars (e.g. Robinson et al.,1997). Under favourable ionospheric conditions it is possible to modify the magnetosphere-ionosphere coupling conditions using the EISCAT Heater. This study involves unmodulated F-region heating during quiet magnetospheric conditions, with the main electrojet currents lying north of Troms $\varnothing$. The action of the heater is, thus, restricted to increasing the backscatter cross section which the F-region ionosphere offers to HF coherent radars. The CUTLASS (Co-operative UK Twin Located Auroral Sounding System) radar is an HF coherent backscatter radar system located at Hankasalmi, Finland and Thykkvibær, Iceland, and forms part of the SuperDARN array (Greenwald et al., 1995). CUTLASS is ideally situated for making observations of the heated volume over Troms $\varnothing$ (e.g. Bond et al., 1997; Eglitis et al., 1998), as shown schematically in Fig. 1. Here data is presented from the SP-UK-OUCH (Observations of ULF waves with CUTLASS and the Heater; Wright and Yeoman, 1999) experiment. In this experiment the CUTLASS radars run in a high temporal and spatial resolution mode, with Hankasalmi running a 6-beam scan (scanning beams 7 through to 2, inclusive) with an integration time of $1 \mathrm{~s}$, whilst Thykkvibær runs a 3-beam scan (beams 13-15) with a $2 \mathrm{~s}$ integration period. Thus, both radars produce data with a temporal resolution of $6 \mathrm{~s}$. Both run in a high spatial resolution mode, with each radar having 75 range gates of $15 \mathrm{~km}$ length, centered on the heated volume at Troms $\emptyset$. For the interval discussed in this paper, only data from Hankasalmi was available for analysis. The EISCAT heater was in continuous operation at $50 \%$ power (using $6 \times 80 \mathrm{~kW}$ transmitters, an ERP of $\sim 130 \mathrm{MW}$ ), at a frequency of $\approx 4 \mathrm{MHz}$ for 4-hour intervals. The heater produces artificial electron density irregularities in the F-region ionosphere which act as targets for the HF radar. The artificial targets have been demonstrated to track accurately the natural ionospheric convection velocity, with the backscatter obtained being of very high power and very narrow spectral width. This allows a short integration time to be run on the radar, providing higher time resolution than is normally available.

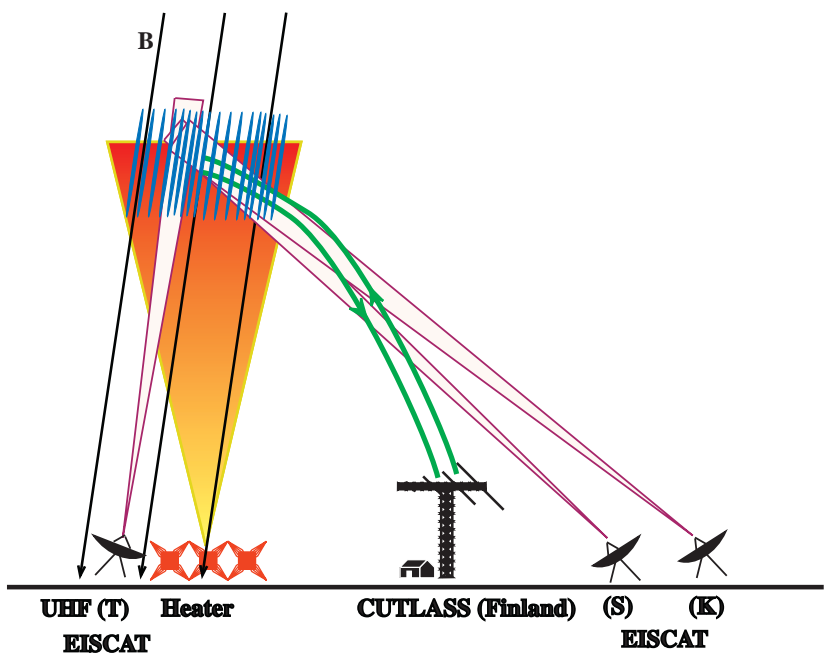

Fig. 1. A schematic of the artificial backscatter experiment, SP-UKOUCH. The Troms $\emptyset$ heater continuously heats the $F$-region ionosphere, creating artificial ionospheric irregularities. Both the CUTLASS HF coherent backscatter radars, operating in a high temporal and spatial resolution mode, detect these irregularities. Measurements are also available from the EISCAT UHF incoherent scatter radar.

\subsection{EISCAT UHF}

The SP-UK-OUCH experiment on 26 October 1999 included operation of the EISCAT UHF incoherent scatter radar (e.g. Rishbeth and Williams, 1985). The beam from the UHF transmit/receive antenna at Troms $\varnothing$, Norway, was pointed along the local magnetic field direction, which is at a geographic azimuth of $183.2^{\circ}$ and an elevation angle of $77.2^{\circ}$. The transmitted pulse schemes include a $350 \mu$ s pulse which provides measurements at Troms $\varnothing$ of electron density, electron temperature, ion temperature and line-of-sight ion velocity at an altitude resolution along the magnetic field direction of some $22 \mathrm{~km}$, extending over 21 range gates from approximately 140 to $600 \mathrm{~km}$ altitude. Two remote site receivers, located at Kiruna in Sweden and Sodankylä in Finland, were directed such that they intersected the transmitter beam at the F-region altitude of $250 \mathrm{~km}$. UHF operation also includes the transmission of a $21 \mu$ s power profile pulse which yields estimates of raw electron density from some 60 to $340 \mathrm{~km}$ altitude at a resolution close to $3.1 \mathrm{~km}$.

\subsection{Polar CAMMICE}

The Polar spacecraft was launched on 26 February 1996 with the objective of making both high- and low-altitude measurements of the Earth's polar regions. The spacecraft describes a highly elliptical orbit, with apogee at 9 Earth radii $\left(R_{E}\right)$, perigee at $1.8 R_{E}$ geocentric and an inclination of $86^{\circ}$. The orbital period is about $18 \mathrm{~h}$. Initially, apogee was over the northern polar region but has been moving towards the equator at about $16^{\circ}$ per year. 


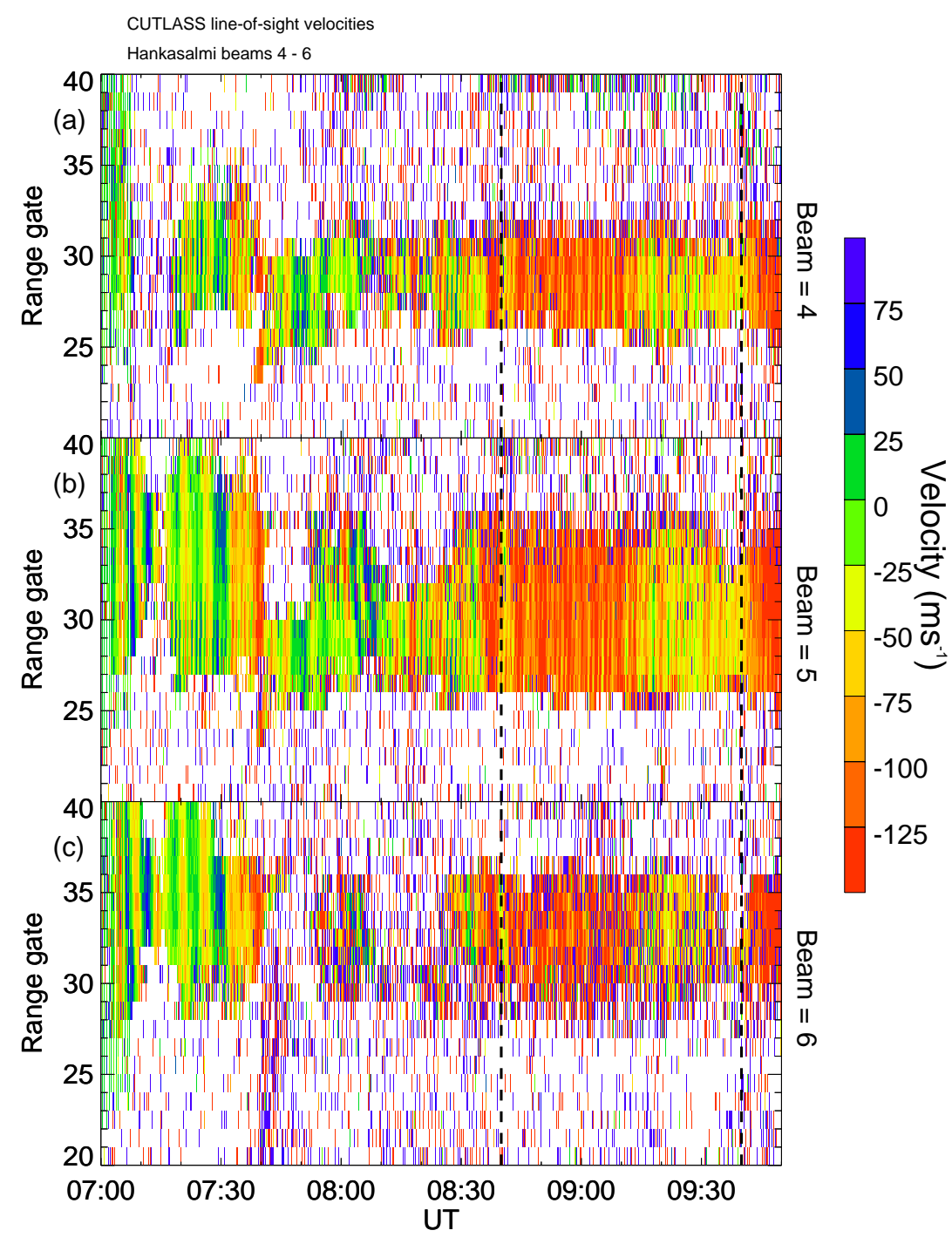

Fig. 2. An overview of the CUTLASS line-of-sight velocity data for the SP-UK-OUCH experiment on 26 October 1999. The experiment ran from 07:00-10:00 UT. The data shown are beams 4,5 and 6 (panels a, b and c, respectively) velocities of the Hankasalmi, Finland radar as a function of range gate. The velocities are colourcoded such that flow away from the radar (negative velocities) are indicated in red and flow toward the radar (positive velocities) are indicated in blue. The interval of data indicated with dashed vertical lines will be examined in detail in this paper.
Data from the MICS (Magnetospheric Ion Composition Sensor; e.g. Wilken et al., 1992) of the CAMMICE (Charge And Mass Magnetospheric Ion Composition Experiment) instrument is utilized for this study. It measures the charge and mass composition of ions within the Earth's magnetosphere over the energy range 1-240 keV/q and provides a full characterization of incident ions by utilizing time-of-flight analysis and energy spectroscopy in combination with an electrostatic entrance filter to measure the mass, energy and ionic charge, with a narrow angle of acceptance allowing determination of the pitch-angle distribution.

The TIMAS (Toroidal Imaging Mass-Angle Spectrograph, Shelley et al., 1995) instrument on the Polar spacecraft is a first order double focusing (angle and energy), imaging spectrograph that simultaneously measures all mass per charge components from $1 \mathrm{AMU} / \mathrm{e}$ to greater than $32 \mathrm{AMU} / \mathrm{e}$ over a nearly $360^{\circ}$ by $10^{\circ}$ instantaneous field-of-view. Mass per charge is dispersed radially on an annular microchannel plate detector and the azimuthal position on the detector is a map of the instantaneous $360^{\circ}$ field-of-view. With the rotation of the spacecraft, the TIMAS sweeps out very nearly a $4 \pi$ solid angle image in a half spin period, where the nominal spin period is $\sim 6 \mathrm{~s}$. The energy per charge range, from $15 \mathrm{eV} / \mathrm{e}$ to $32 \mathrm{keV} / \mathrm{e}$, is covered in 28 steps. Each energy step is sampled for approximately $20 \mathrm{~ms} ; 14$ step (odd or even numbered) energy sweeps are completed 16 times per spin. Further details of the TIMAS instrument can be found in Shelley et al. (1995).

\section{Observations}

\subsection{CUTLASS}

During the experimental run of the SP-UK-OUCH, the artificial backscatter technique provided unprecedented spatial, temporal and electric field resolution. Figure 2 presents a colour-coded representation (flow away from the radars (negative velocities) are colour-coded red, with flow towards the radars (positive velocities) colour-coded blue) of the 
1-o-s (line-of-sight) velocity measured by the CUTLASS Hankasalmi radar for radar range gates 20 through to 40 . The experiment ran from 07:00-10:00 UT. The data shown are Beams 4, 5 and 6 (panels a, b and c, respectively) velocities of the Hankasalmi, Finland radar as a function of time. The EISCAT heater at Troms $\varnothing$ illuminates a region of the high-latitude ionosphere of roughly one degree in latitude.

Although there are other, earlier, wave features observed in the data, this study focuses only on the later part of the interval from 08:40 to 09:40 UT. This is due to the Polar spacecraft being conjugate with Troms $\varnothing$ at that time and, thus, the corresponding magnetospheric particle data for the later wave interval has been obtained. The low frequency wave observed from 07:00 to 07:30 UT in beams 5 and 6 is of a large scale size, with an analysis of data from the IMAGE magnetometer array indicating a clear ground signature over this time period. Thus, it is concluded that the earlier wave is most likely to be the result of a field line resonance or impulsive event with an energy source external to the magnetosphere. The details of this wave interval cannot be determined more precisely due to the limited number of wave cycles available and the complex nature of the wave activity. By 07:18 UT, clear evidence of a higher frequency wave, of the same nature as the event studied in detail here, can be seen in the lower latitude range cells of the artificial scatter region. IMAGE magnetometer data were examined also for the time period 08:40-09:40 UT, when the majority of high frequency wave activity was observed in the ionosphere by the CUTLASS radar. Although some evidence is seen of the earlier wave activity, from 07:00 to 07:30 UT, in the magnetometer data as mentioned above, no conclusive evidence of the high frequency wave was viewed on the ground.

\subsection{EISCAT}

Figure 3 presents the measurements taken by the EISCAT UHF radar at Troms $\emptyset$. The top panel illustrates a timeseries representation of CUTLASS velocities from beam 5 of the Hamkasalmi radar, ranges 27-32. Within the volume of intersection of the three UHF receiver beams, the three measured components of line-of-sight ion velocity can be combined to provide the 3-dimensional ion velocity vector. In this case, the vector velocity, calculated at a temporal resolution of $15 \mathrm{~s}$, has been resolved into components parallel and perpendicular to the local magnetic field direction. The field-perpendicular ion velocity has been further resolved into its component along and perpendicular to the direction of beam 5 of the CUTLASS Finland radar within range gate 28, this being the Finland range cell which corresponds magnetically to the UHF observation volume. These velocity components are illustrated in the bottom panel of Fig. 3. The parallel velocity is superimposed in red on the top panel to allow a direct comparison with CUTLASS measurements. Complex and variable wave activity is clear in data from both radars, with a high level of agreement between CUTLASS Hankasalmi and EISCAT.

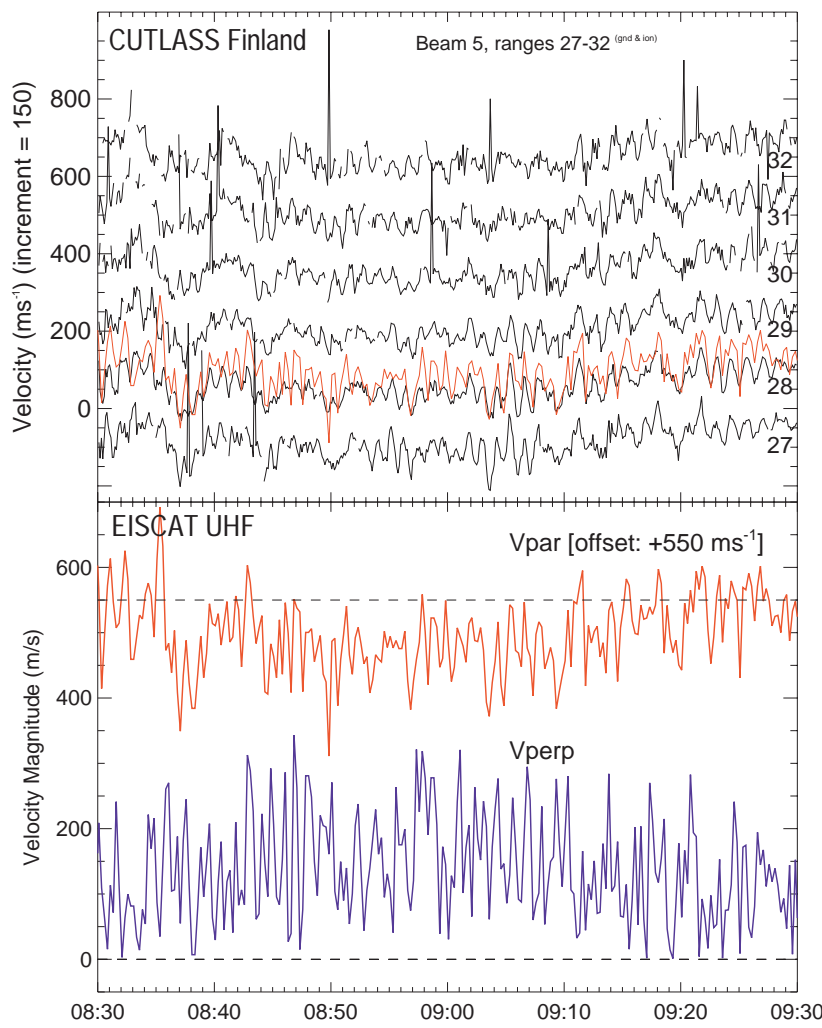

Fig. 3. The top panel illustrates CUTLASS velocities from beam 5 of the Hankasalmi radar, ranges 27-32. The bottom panel illustrates the two field perpendicular components of the EISCAT radar tristatic velocity measurements, resolved parallel and perpendicular to the direction of CUTLASS beam 5, range gate 28. The parallel velocity is superimposed in red on the top panel to allow a direct comparison with CUTLASS measurements of the wave event shown in Figs. 2 and 3, using beam 5, range gate 28 for the interval 08:30-09:30 UT.

A Fourier analysis of spectral power and phase of the wave over several different time periods was undertaken. The wave was analyzed between 08:50-09:00 UT, 09:01-09:08 UT and 09:21-09:28 UT. Figure 4a shows a Fourier power spectrum of the wave; observed in the CUTLASS Hankasalmi velocity data from beam 5, range gate 28 taken from 09:21 to 09:28 UT. The dominant Fourier component is at $13.9 \mathrm{mHz}$, although there is some evidence of wave activity at a lower frequency centered around $3 \mathrm{mHz}$. The analysis from the other time periods indicated how and if the wave altered frequency during the observations. The dominant Fourier components during these periods were $15.8 \mathrm{mHz}, 16.6 \mathrm{mHz}$ and $13.9 \mathrm{mHz}$, respectively. Again, there is some evidence of wave activity around $3 \mathrm{mHz}$. Given this slight modulation in dominant frequency of the wave, an average value of $15.4 \mathrm{mHz}$ was used when considering the resonance equation.

The magnetic latitude profile of the peak Fourier power and phase deduced from the radar data at $13.9 \mathrm{mHz}$ is shown in Fig. 4b. The wave shows some evidence of poleward phase propagation, with the amplitude peak located towards 
(a)

Power Spectrum

Hankasalmi Beam 5, Gate 28

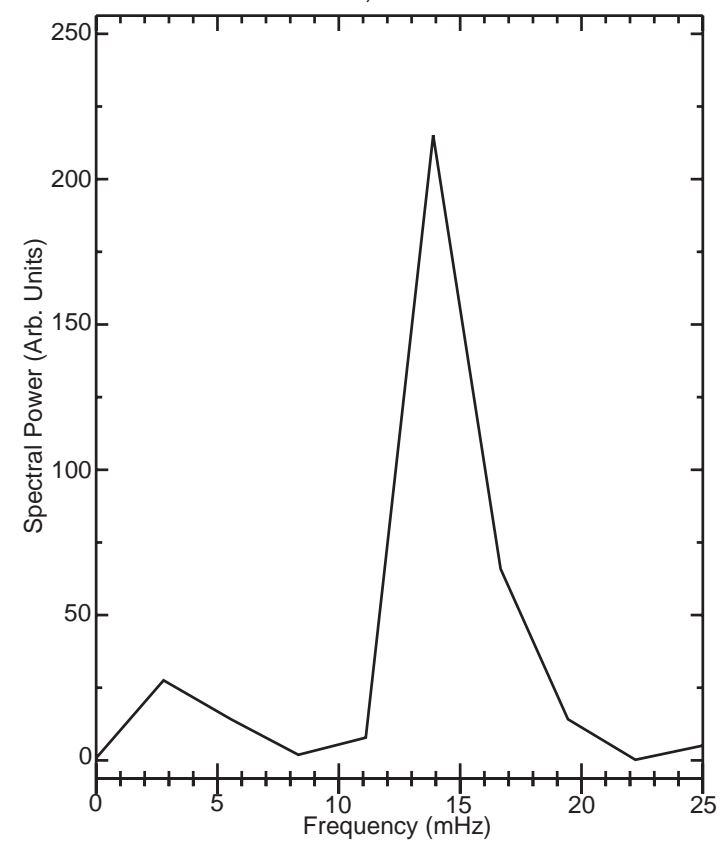

(b)

Hankasalmi beam 5 I-o-s velocity -

Fourier amplitude and phase vs. latitude $(13.89 \mathrm{mHz})$

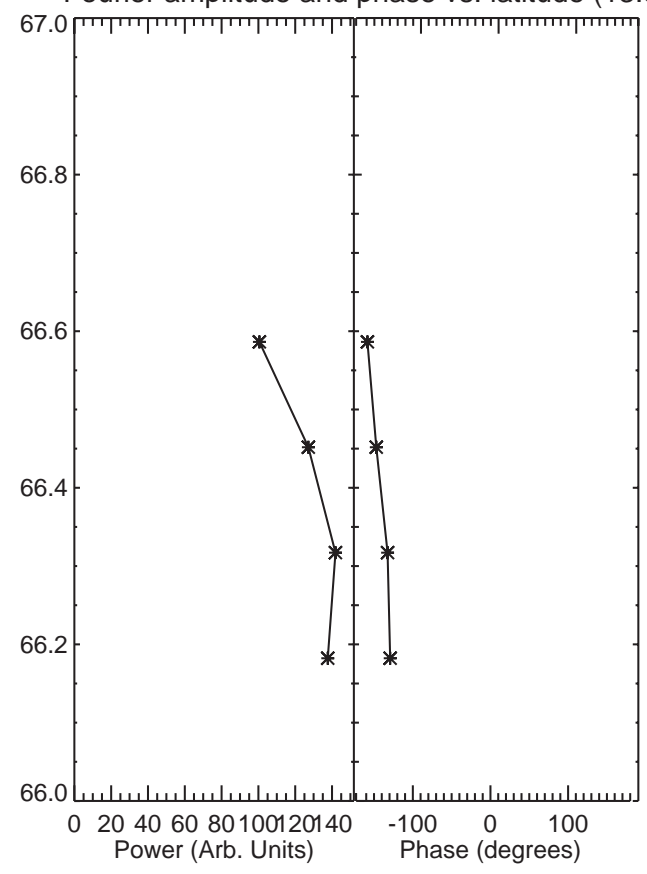

Fig. 4. (a) Fourier power spectrum, (b) magnetic latitude profile of Fourier power and phase at $13.9 \mathrm{mHz}$ over the same interval.

the equatorward edge of the artificial scatter region, close to $66.3^{\circ}$ magnetic latitude. Only latitudes poleward of the resonance location are within the artificial scatter region; this, presumably, precludes the observation of the full $180^{\circ}$ phase change expected around resonance (e.g. Walker et al., 1979). It is noted, however, that while studies by Allan et al. (1982,
1983) showed that stormtime Pc5 waves do not have an associated $180^{\circ}$ change of phase, work by Chisham et al. (1992) showed that there is one associated with $P g \mathrm{~s}$; thus it is unclear whether or not one is expected for this event. A comparison of beams 4 and 5 of the Hankasalmi radar, which at the same magnetic latitude are separated in azimuth, reveal that the wave has an azimuthal wave number, $m=-45 \pm 10$. The negative sign indicates that the wave displayed a westward phase propagation, in the same direction as gradientcurvature drifting protons.

An examination of estimates of the ionospheric Hall and Pedersen conductivities, as derived from the EISCAT radar, shows no significant modulations at the wave frequency for this event. Hence, this small amplitude event does not modulate the ionospheric conductivity, unlike the large amplitude events studied by Buchert et al. (1999) and Lester et al. (2000), which showed large ground magnetic field oscillations and accompanying conductivity modulations.

\subsection{CAMMICE}

Figure 5a defines the orbit of the Polar spacecraft, in SM coordinates. The specific interval studied in this paper is highlighted in grey. The footprint of the spacecraft mapped magnetically to Troms $\emptyset$ at a time of 09:05 UT. Its onboard instrumentation includes energetic particle detectors and magnetometers.

Figures $5 \mathrm{~b}$ and $\mathrm{c}$ show two particle distributions as measured by the CAMMICE (MICS) energetic particle instrument onboard Polar, between a time of 09:00 and 09:10. The figures represent the ion distribution functions in phase space as a function of particle energy and clearly demonstrate that an unstable (non-Maxwellian) population of protons, with a positive gradient $(\partial f / \partial W>0)$ in the energy range of 8 to $15 \mathrm{keV}$, was observed on, or near the Troms $\varnothing$ fieldline. These measurements were made at a local time of 11:25 MLT as the spacecraft travelled southward in the Northern Hemisphere at an L-shell location of $\sim 6$. As mentioned earlier, this 'bumpon-the-tail' distribution implies that free energy is available to the wave if the drift-bounce resonance equation is satisfied at these energies.

\section{Discussion}

The wave presented in this paper was observed to occur in the morning sector, in artificially created field-aligned irregularities generated by the EISCAT heating facility located at Troms $\varnothing$. Through an FFT analysis of line-of-sight velocity measurements, made by the CUTLASS radar located at Hankasalmi, the wave was shown to have a dominant frequency of $\approx 15.4 \mathrm{mHz}$. A comparison of beams 4 and 5 of the Hankasalmi radar, which are separated in azimuth, but at the same magnetic latitude, reveal that the wave has an azimuthal wave number, $m=-45 \pm 10$. The characteristics of the wave are similar in nature to that of a $P g$; however, the magnitude of this wave is not sufficient enough for it to be 
POLAR orbit 1999298 (10/25) 18:57 UT to 1999299 (10/26) 13:12 UT

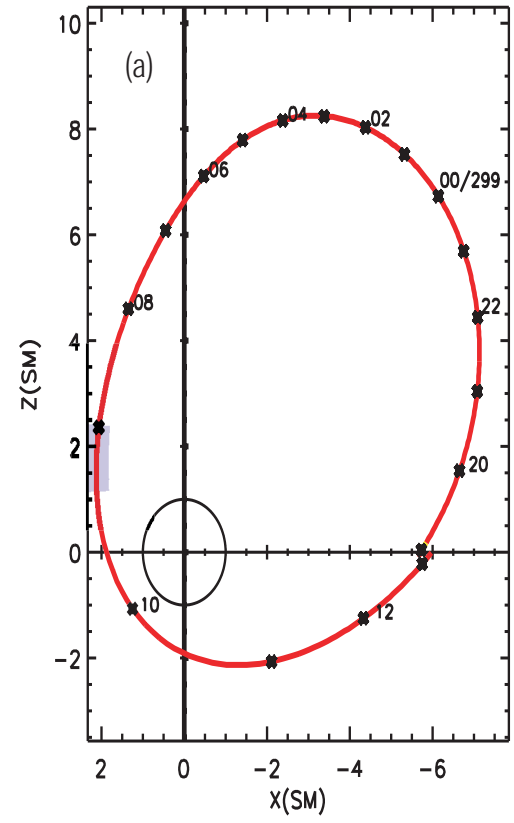

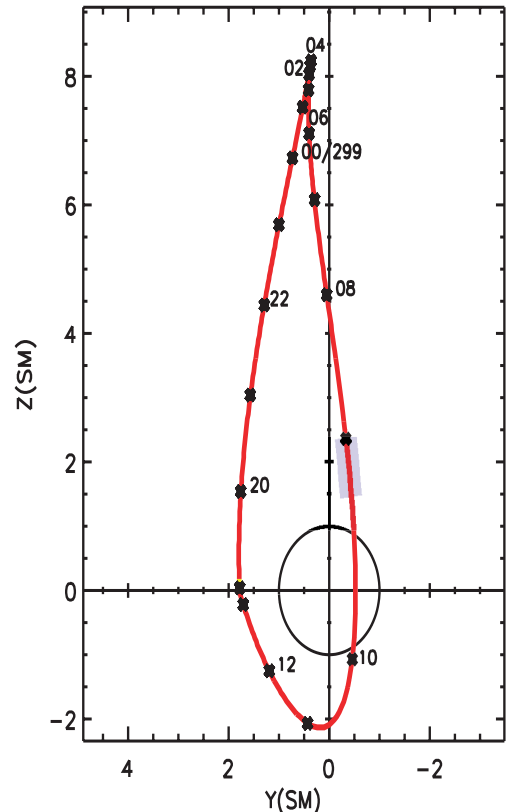

Polar CAMMICE (MICS) ION DISTRIBUTION FUNCTIONS (IDFs)

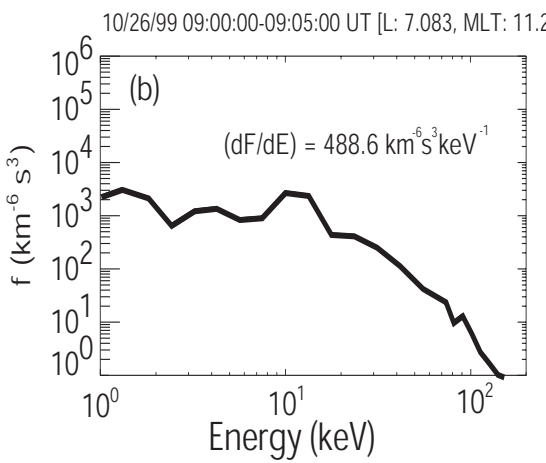

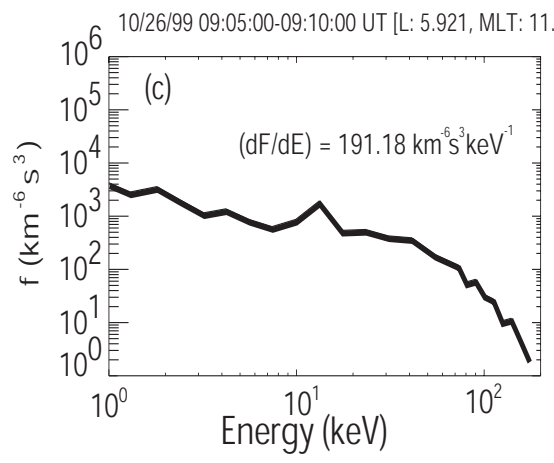

Fig. 5. (a) A section of the orbit described by the Polar spacecraft on 26 October 1999 expressed in a Solar Magnetic coordinate frame. The shaded grey region indicates the interval where the ion distribution functions were derived from the onboard particle detector CAMMICE (MICS). (b) (c) Ion distribution functions, $f$, derived from the CAMMICE (MICS) instrument for the intervals (a) 09:0009:05 UT and (b) 09:05-09:10 UT. During this time the spacecraft traversed L-shells 7.1-5.9 and 11:26-11:21 MLT. detected on the ground. The negative sign of the $m$ number indicates that the wave has phase propagation in the direction of gradient-curvature drifting protons that have been injected into the inner magnetosphere on the nightside.

Polar magnetic field observations (not shown) reveal a short-lived wave packet to exist as the spacecraft crosses the Troms $\varnothing$ field line. The frequency of the observed wave is consistent with the ground observations, although the shortlived nature of the observations precludes a more detailed comparison.

If the drift-bounce resonance condition, Eq. (1), is considered, knowing both the angular frequency and azimuthal wave number of the wave, $m$, certain constraints can be added to the drift and bounce angular frequencies and, hence, the energies of the particle population thought to be driving the wave. By examining both sides of the resonance equation independently, it becomes possible to see if this wave could be due either to a drift $(N=0)$ or drift-bounce $(N=1)$ resonance interaction.

The expressions below quantify the drift (Chisham, 1996) and bounce (Glassmeier et al., 1999; Schulz and Lanzerotti, 1974) angular frequencies in the Earth's frame of reference as a function of proton energy.

$$
\begin{aligned}
\omega_{d}= & -\frac{6 W L(0.35+0.15 \sin \alpha)}{B_{S} R_{E}^{2}} \\
& +\frac{90\left(1-0.159 K_{p}+0.0093 K_{p}^{2}\right)^{-3} L^{3} \sin \varphi}{B_{s} R_{E}^{2}} \\
\omega_{d}= & -\frac{6 W L(0.35+0.15 \sin \alpha)}{B_{S} R_{E}^{2}}+\frac{E L^{2}}{B_{s} R_{E}} \\
\omega_{b}= & \frac{\pi \sqrt{W}}{\sqrt{2 m_{p}} L R_{E}(1.3-0.56 \sin \alpha)}
\end{aligned}
$$

where $W$ is the proton energy in $\mathrm{eV}, L$ is the proton's $\mathrm{L}$-shell, $B_{S}$ is the equatorial surface magnetic field strength, $R_{E}$ is the 


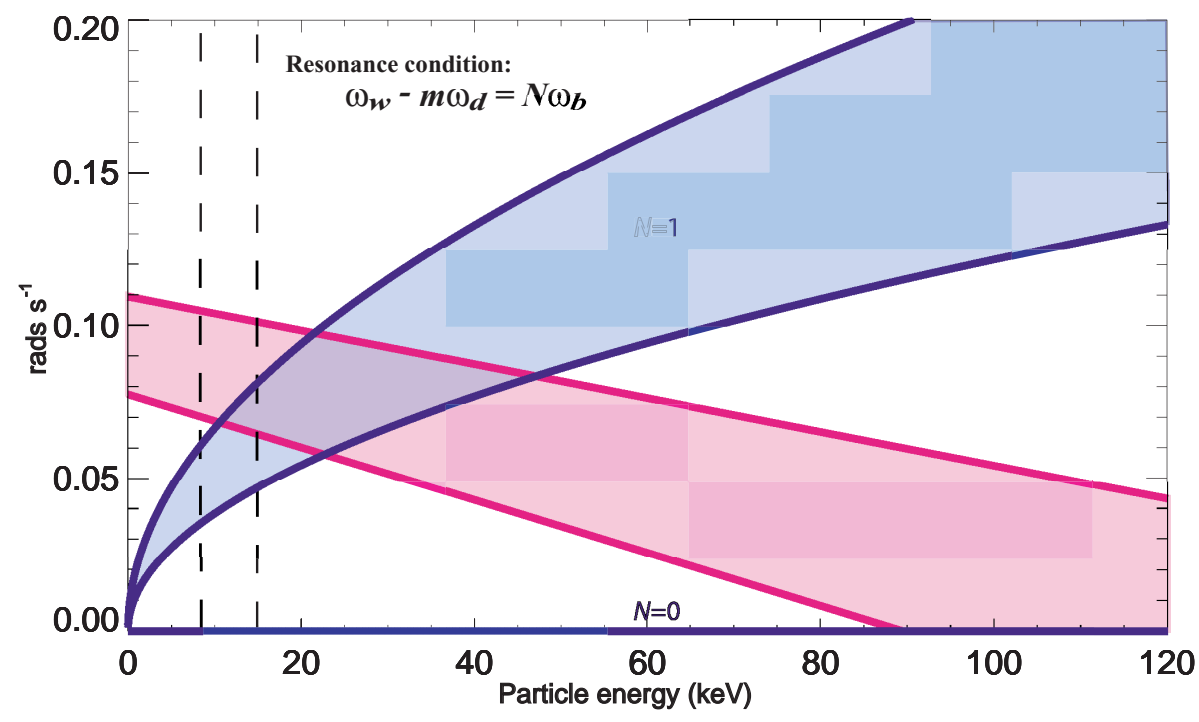

Fig. 6. A graphical representation of the drift-bounce resonance condition (see text for details). It can be seen that the two shaded areas intersect at two locations, with these locations revealing the energies at which the resonance condition is satisfied. If free energy is available to the wave at these interaction energies in the form of a positive gradient in the IDF, then the particle population can provide energy for that wave mode. The dashed vertical lines represent the upper and lower particle energies of the positive gradient $(\partial f / \partial W>0)$ as measured by the CAMMICE (MICS) instrument onboard the Polar spacecraft. radius of the Earth, $\alpha$ is the proton equatorial pitch-angle, $\varphi$ the azimuth of the particle measured anticlockwise from local midnight, $K_{p}$ the planetary magnetic activity index and $m_{p}$ the proton mass. The first term on the right-hand side of Eq. (2) represents gradient-curvature drift (a typical value of $-1.44 \times 10^{-4} \mathrm{rads} / \mathrm{s}$ is obtained for a $10 \mathrm{keV}$ proton) and the second $\boldsymbol{E} \wedge \boldsymbol{B}$ drift under a model magnetospheric potential (a value of $7.7 \times 10^{-6} \mathrm{rads} / \mathrm{s}$ is obtained here). The actual magnetospheric electric field can be estimated from the ionospheric electric field as measured by radar, if it is available. Under these circumstances, Eq. (2) may be replaced with Eq. (3), where $E$ is the magnetospheric electric field. In this study, a value of North-South model magnetospheric potential was used after estimates of the measured ionospheric electric field $(5 \mathrm{mV} / \mathrm{m})$ were found to imply a drift speed of a similar magnitude; this has little impact, given the error ranges of the calculations discussed below. The magnetosphere was magnetically quiet during the interval, so a value of $K_{p}=2$ was used in the equations. For a $10 \mathrm{keV}$ particle a total drift angular frequency of $-1.36 \times 10^{-4} \mathrm{rads} / \mathrm{s}$ is obtained. By substituting these values into Eq. (1) it is possible to ascertain whether a drift or drift-bounce resonance was the likely driving mechanism of the wave.

The results are shown in graphical form in Fig. 6. The plot indicates at what energies the drift resonance and driftbounce resonance condition are satisfied, with the $x$-axis representing the energy of the particle population in $\mathrm{keV}$ and the $y$-axis representing the right-hand side and left-hand side of Eq. (1) in units of radians per second. The blue areas indicate the right-hand of the resonance equation, which deals with the contribution from the bounce angular frequency of the particles. Shown on the graph are the conditions for the $N=0$, drift resonance and the $N=1$, drift-bounce resonance condition. The pink area represents the left-hand side of the resonance equation, which takes into account the angular frequency of the wave, the drift angular frequency of the particles and the azimuthal wave number of the wave, $m$. The spread in both the pink and blue lines represents the maximum errors brought about due to uncertainties in the parameters used to obtain values for $\omega_{d}, \omega_{b}, m$ and $\omega_{\text {wave }}$. Changes in the spacecraft location during the wave event introduce errors in both $L$ and $\varphi$. The largest errors come from the measurement of the angular frequency and m number of the wave and the value for particle pitch-angle. As mentioned in earlier sections, the wave frequency does alter slightly during the period studied. When considering the resonance equation, values of the maximum and minimum frequency were used to ensure uncertainties were included. The $m$ value of the wave also carries an error of \pm 10 . Another source of error is brought about when considering the pitch-angle distribution of the particle population. As discussed in Southwood and Kivelson (1982), drift resonance is expected to occur primarily with particles of large pitch angles in the equatorial plane and drift-bounce resonance with particles of smaller pitch-angles. The IDFs presented in Fig. 5 are not strong functions of pitch-angle although few particles of close to field-aligned pitch-angles are observed and omnidirectional IDFs are presented. In the calculations of the particle drift and bounce angular frequencies we use a range of large pitch angles in the drift-frequency calculation, although this range has little impact on the results as the impact of uncertainties in $m$ and $\omega_{\text {wave }}$ are larger. For the drift-bounce angular frequencies we use a range of smaller pitch-angles, as is appropriate for the drift-bounce resonance condition. For this resonance condition, the errors in $\omega_{b}$ are the dominant factor in determining the range of particle energies which satisfy the resonance condition. It can be seen that the lines intersect at two locations with these locations revealing the energies at which the resonance condition is satisfied. If free energy is available to the wave at these interaction energies, in the form of a positive gradient in the IDF, then the particle population can drive that wave mode. From the graph, it can be seen 


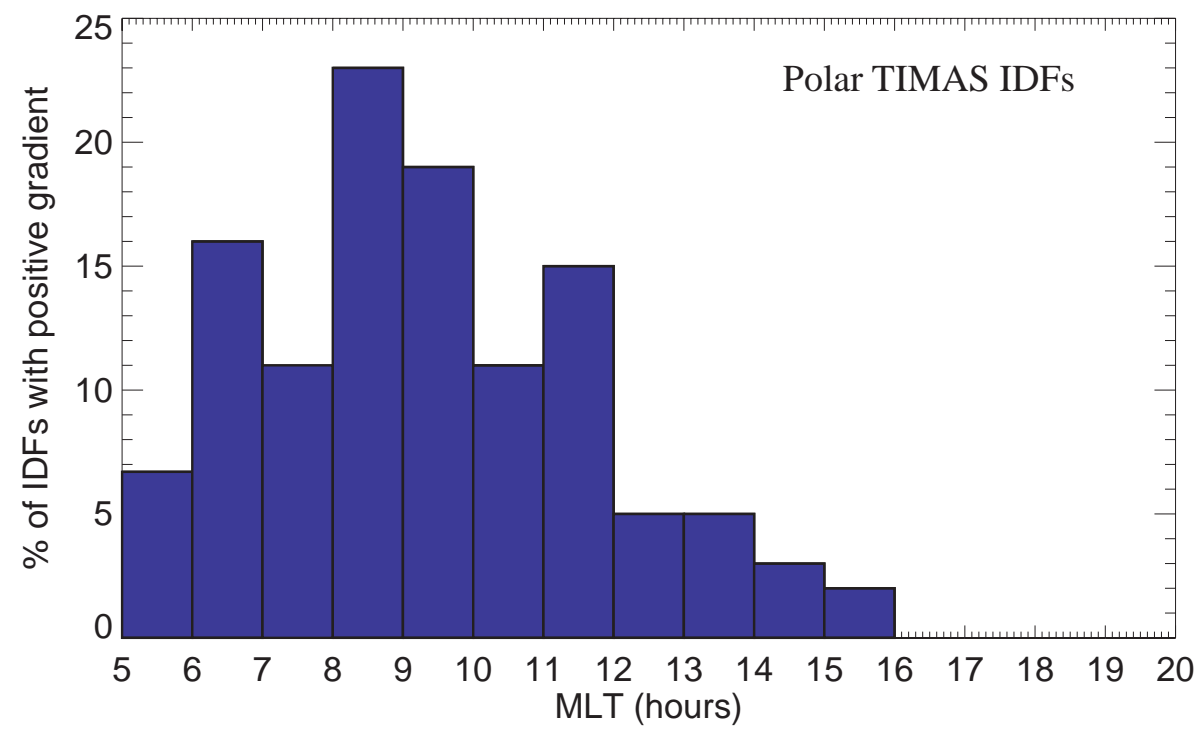

Fig. 7. Results of a statistical analysis of two years worth of Polar TIMAS data from the Troms $\varnothing$ L-shell. The graph indicates the percentage occurrence of IDFs that contain a similar positive gradient over a similar energy range to the IDF seen in the CAMMICE data in Fig. 5, as a function of magnetic local time. that a drift resonance process can be driven at $\sim 110 \mathrm{keV}$ or a drift-bounce resonance interaction at $\sim 10-40 \mathrm{keV}$.

During the wave event, the CAMMICE energetic particle instrument onboard the Polar spacecraft detected a nonMaxwellian population of protons drifting westward in the inner magnetosphere. The measurements were made at 09:05-09:10 UT (a local time of 11:25 MLT) as the spacecraft travelled equatorward through the Northern Hemisphere, crossing the Troms $\emptyset$ field line. The distribution had an energy range of 1-200 keV with a clear bump-on-the-tail evident at an energy of $\sim 8-15 \mathrm{keV}$. These energies overlap the lower end of the predicted drift-bounce resonance condition presented in Fig. 6. It is, thus, clear that the wave observed by both the CUTLASS and EISCAT radars shows evidence of being generated by a drift-bounce resonance interaction between a second harmonic standing wave and a non-Maxwellian energetic particle population with a positive gradient, located between 8 and $15 \mathrm{keV}$, but is not consistent with a drift resonance mechanism.

Further analysis has been undertaken using two years (1996-1998) of data from the Polar TIMAS instrument. Intervals were considered when the spacecraft was located at a magnetic L-shell of between 5 and 8; the orbits covered an MLT range of 06:00-18:00 MLT. Figure 7 describes results obtained in relation to the study of this morning sector wave. The data were binned according to MLT location of the spacecraft. The occurrence of positive gradients in the IDFs was then examined to discover the prevalence of these non-Maxwellian IDFs that contain a positive gradient of a similar magnitude $\left(\sim 300 \mathrm{~km}^{-6} \mathrm{~s}^{3} \mathrm{keV}^{-1}\right)$ and similar energy range $(\sim 8-15 \mathrm{keV})$ to that seen in the CAMMICE data for the wave studied in this paper. As is shown in the figure, the highest percentage occurrence of this was in the morning sector with $\sim 20 \%$ of IDFs showing evidence of a positive gradient between 8 and $15 \mathrm{keV}$. In the afternoon sector, however, this percentage has decreased to less than 5\%. This analy- sis indicates the prevalence of these non-Maxwellian IDFs at this energy range in the morning sector inner magnetosphere.

\section{Relationship with previous observations}

There has been considerable debate as to the exact nature of the interaction mechanisms for such wave particle interactions observed in the morning and afternoon sectors. Previous observations by Yeoman and Wright (2001), from the SP-UK-OUCH campaign, have identified both $\operatorname{drift}(N=0)$ and drift-bounce $(N=1)$ mechanisms as viable candidates for high $m$ waves as observed in the high-latitude ionosphere in the afternoon sector. In that paper, evidence of a $(N=0)$ fundamental mode, which evolves into a $(N=1)$ second harmonic wave mode, is presented. Here, we present evidence of a morning sector $(N=1)$ second harmonic drift-bounce resonance. While the characteristics of the wave discussed in this paper are similar to those observed for $P g \mathrm{~s}$, the amplitude of the wave is not large enough to be observed by ground magnetometers. Such $P g$-like events in the morning sector have been demonstrated to be a common phenomenon (if a difficult one to observe), Yeoman et al. (2000). Wright et al. (2001) presented an example of a particle driven $P g$. They present both data from the ground IMAGE magnetometer array and DOPE as well as conjugate particle data from Polar; they surmise that the wave was the result of wave particle interactions between a non-Maxwellian particle population drifting westwards and a second harmonic standing wave through the drift-bounce $(N=1)$ resonance mechanism, the same interaction thought to be responsible for driving the wave presented in this paper. The azimuthal $m$ number for the $P g$ is estimated to be $-30 \pm 5$. While it is generally agreed that both drift $(N=0)$ and drift-bounce $(N=1)$ mechanisms are evident in the afternoon sector of the magnetosphere, much controversy still surrounds the 
question of which is the dominant mechanism in the morning sector. There are conflicting theories as to the mechanism which drives the dawnside $P g$ s and other high $m$ waves. Previous papers have cited both drift-bounce (second harmonic) resonance (Chisham and Orr, 1991) and drift (fundamental) resonance (Takahashi et al., 1992). A recent paper by Ozeke and Mann (2001) illustrates a model in which they reveal the energetically favourable locations in energy - L-shell space where unstable particle distributions $\partial f / \partial W>0$ ) can occur. The model predicts the location of these particle populations by ascertaining the position where the open-closed orbit boundary of the azimuthally drifting particle population intersects a drift and drift-bounce resonance curve. During geomagnetically active times, closed orbit paths around the Earth become open and energetic particles can be injected into them and to the inner magnetosphere. If a period of low geomagnetic activity follows, these paths become closed once more and the newly injected particles can drift round the Earth to the morning sector from their injection point on the nightside. In the morning sector, they will, on occasion, match the local drift-bounce resonance condition. In their model, Ozeke and Mann (2001) show that only the $N=1$ resonance curve crosses the open-closed westward orbit boundary in the morning sector. Thus, they conclude that only the $N=1$ resonance is a viable candidate to be driven by bump-on-the-tail distributions. The morning sector wave presented in this paper is concluded to have similarly been brought about through the drift-bounce $(N=1)$ resonance interaction mechanism. Their model, therefore, is in good agreement with the findings of this paper.

At the low energy end of the range, where $\partial f / \partial W>0$, the drifting protons would have been strongly influenced by the dawn-dusk magnetospheric electric field. Due to the unpredictability of the drift paths of the protons under these conditions, it is very difficult to be able to identify an individual substorm particle injection event as a source of these particles. In fact, the energies are so low that the protons may not be the result of a substorm; rather the unstable population could be the result of other magnetospheric processes. The magnetosphere was geomagnetically quiet for several hours before the wave on the 26 October 1999 was observed (an index of $K_{p}=2$ was measured). Thus, it is reasonable to hypothesise that the protons thought to be driving the wave are of low enough energy that they could have drifted, in the ring current, from the nightside to the morning sector without being scattered out of the magnetosphere, again consistent with the model of Ozeke and Mann (2001).

\section{Summary}

Data from both the CUTLASS HF radars and the EISCAT UHF radar have been analyzed to investigate ULF wave processes observed in the morning sector of the inner magnetosphere. HF radar backscatter, which has been artificiallyinduced by the high power RF facility at Troms $\varnothing$, provided ionospheric electric field data of unprecedented temporal res- olution and accuracy. Data from the SP-UK-OUCH experiment have revealed small-scale waves with an azimuthal wave number, $m \approx-45 \pm 10$, in the morning sector. An FFT analysis of the data revealed the angular frequency of the wave to be $\approx 15.4 \mathrm{mHz}$. Conjugate observations from the Polar CAMMICE instrument indicate the presence of a non-Maxwellian IDF, with evidence that free energy was available to the wave between 8 and $15 \mathrm{keV}$. An analysis of the drift-bounce resonance equation shows that the wave observed was the result of a drift-bounce $(N=1)$ resonance interaction between a second harmonic standing wave and the non-Maxwellian particle population. Further statistical analysis was undertaken, using the Polar TIMAS instrument, to reveal the prevalence and magnitude of the non-Maxwellian energetic particle populations thought to be responsible for generating these wave types. It was seen that $\sim 20 \%$ of all IDFs contain a positive gradient over a similar energy range to that seen in the data for the wave discussed here, in the morning sector only. The evidence presented suggests that the drift-bounce resonance condition, with particle populations of relatively low energy $(\sim 10 \mathrm{keV})$, is the most likely mechanism for the bulk of such high $\mathrm{m}$ morning sector waves. A statistical analysis of TIMAS data is underway with the aim of discovering how prevalent non-Maxwellian IDFs are in both the morning and evening sectors of the magnetosphere, in addition to quantifying the magnitude and energy of the 'bump-on-the-tail' events. The aim is to combine this statistical data base with ground based measurements to obtain evidence of the exact nature of the energy generation mechanism for these high $m$ waves observed in both the morning and afternoon sector magnetosphere.

Acknowledgements. The authors thank the director and staff of EISCAT for the operation of the Troms $\emptyset$ heater facility. EISCAT is an international facility funded collaboratively by the research councils of Finland (SA), France (CNRS), the Federal Republic of Germany (MPG), Japan (NIPR), Norway (NAVF), Sweden (NFR) and the United Kingdom (PPARC). The SuperDARN Hankasalmi and Thykkvibær HF radars are deployed and operated by the University of Leicester and funded by the PPARC (Grant number PPA/R/R/1997/00256), the Finnish Meteorological Institute and the Swedish Institute for Space Physics. LJB is supported by a PPARC Quota Studentship. We would also like to thank Prof. Theodore Fritz, PI of the CAMMICE (MICS) instrument, for supplying data; Dr William Peterson, PI of the TIMAS instrument and Dr. I. J. Rae for his assistance in obtaining the Polar data.

The Editor in Chief thanks W. Allen and S. Buchert for their help in evaluating this paper.

\section{References}

Allan, W., Poulter, E. M., and Nielsen, E.: STARE observations of a Pc5 pulsation with large azimuthal wave number, J. Geophys. Res. 87, 6163, 1982.

Allan, W., Poulter, E. M., and Nielsen, E.: Pc5 pulsations associated with ring current proton drifts: STARE radar observations, Planet. Space Sci., 31, 1279, 1983.

Anderson, B. J., Engebretson, M. J., Rounds, S. P., Zanetti, L. J., and Potemra, T. A.: A statistical study of Pc 3-5 pulsations ob- 
served by the AMPTE/CCE magnetic fields experiment. 1. Occurrence distributions, J. Geophys. Res., 95, 10 495, 1990.

Bond, G. E., Robinson, T. R., Eglitis, P., Wright, D. M., Stocker, A. J., Rietveld, M. T., and Jones, T. B.: Spatial observations by the CUTLASS coherent scatter radar of ionospheric modification by high power radio waves, Ann. Geophysicae., 15, 1412, 1997.

Buchert, S. C., Fujii, R., and Glassmeier, K. H.: Ionospheric conductivity modulations in ULF pulsations, J. Geophys. Res., 104, $10119,1999$.

Chisham, G.: Giant pulsations: An explanation for their rarity and occurrence during geomagnetically quiet times, J. Geophys. Res., 101, 24 755, 1996.

Chisham, G. and Orr, D.: Statistical studies of giant pulsations (Pgs): harmonic mode, Planet. Space Sci., 39, 999, 1991

Chisham, G., Orr, D., and Yeoman, T. K.: Observations of a giant pulsation $(\mathrm{Pg})$ across an extended array of ground magnetometers and an auroral radar, Planet. Space Sci., 40, 953, 1992.

Eglitis, P., Robinson, T. R., Rietveld, M. T., Wright, D. M., and Bond, G. E.: The phase speed of artificial irregularities observed by CUTLASS during HF modification of the auroral ionosphere, J. Geophys. Res., 103, 2253, 1998.

Engebretson, M. J., Murr, D. L., Erickson, K. N., Strangeway, R. J., Klumpar, D. M., Fuselier, S. A., Zanetti, L. J., and Potemra, T. A.: The spatial extent of radial magnetic pulsation events observed in the dayside near synchronous orbit, J. Geophys. Res., 97, $13741,1992$.

Glassmeier, K. H.: Reply to the comment by I. R. Mann and G. Chisham, Ann. Geophysicae, 18, 167, 2000.

Glassmeier, K. H., Buchert, S., Motschmann, U., Korth, A., and Pedersen, A.: Concerning the generation of geomagnetic giant pulsations by drift-bounce resonance ring current instabilities, Ann. Geophysicae, 17, 338, 1999

Grant, I. F., McDiarmid, D. R., and McNamara, A. G.: A class of high-m pulsations and its auroral radar signature, J. Geophys. Res., 97, 8439, 1992.

Greenwald, R. A., Weiss, W., Nielsen, E., and Thompson, N. R.: STARE: A new radar auroral backscatter experiment in northern Scandinavia, Radio Sci., 13, 1021, 1978.

Greenwald, R. A., Baker, K. B., Dudeney, J. R., et al.: DARN/SUPERDARN A global view of the dynamics of highlatitude convection, Space Sci. Rev., 71, 761, 1995.

Hughes, W. J.: Hydromagnetic waves in the magnetosphere, Solar Terrestrial Physics (edited by Carovillano, R. L. and Forbes, J. M.), Reidel, Dordrecht, 1983.

Hughes, W. J. and Southwood, D. J.: The screening of micropulsation signals by the atmosphere and ionosphere, J. Geophys. Res., $81,3234,1976$.

Hughes, W. J., McPherron, R. L., Barfield, J. N., and Mauk, B. H.: A compressional Pc4 pulsation observed by three satellites in geostationary orbit near local midnight, Planet. Space Sci., 27, 821,1979

Hughes, W. J., Southwood, D. J., Mauk, B., McPherron, R. L., and Barfield, J. N.: Alfvén waves generated by an inverted plasma energy distribution, Nature, 275, 43, 1978.

Kokubun, S.: Statistical characteristics of Pc5 waves at geostationary orbit, J. Geomag. Geoelectr., 37, 759, 1985.

Lessard, M. R., Hudson, M. K., and Lühr, H.: A statistical study of Pc 3-Pc 5 magnetic pulsations observed by the AMPTE/Ion Release Module satellite, J. Geophys. Res., 104, 4523, 1999.

Lester, M., Davies, J. A., and Yeoman, T. K.: The ionospheric response during an interval of Pc5 ULF wave activity, Annales Geophysicae, 18, 257, 2000.
Mann, I. R. and Chisham, G.: Comment on 'Concerning the generation of geomagnetic giant pulsations by drift-bounce resonance ring current instabilities' by K. H. Glassmeier et al., in Ann. Geophysicae, 17, 338, 1999, Ann. Geophysicae, 18, 161, 2000.

McNamara, A. G., McDiarmid, D. R., Sofko, G. J., Koeler, J. A., Forsyth, P. A., and Moorcroft, D. R.: BARS - a dual bistatic auroral radar system for the study of electric fields in the Canadian sector of the auroral zone, Adv. Space Res., 2, 145, 1983.

Nielsen, E., Guttler, W., Thomas, E. C., Stewart, C. P., Jones, T. B., and Hedburg, A.: SABRE -new radar-auroral backscatter experiment, Nature, 304, 712, 1983.

Ozeke, L. G. and Mann, I. R.: Modeling the properties of high $\mathrm{m}$ Alfvén waves driven by the drift-bounce resonance mechanism, J. Geophys. Res., 106, 15 583, 2001.

Rietveld, M. T., Kohl, H., Kopka, H., and Stubbe, P.: Introduction to ionospheric heating at Troms $\emptyset-$ I. Experimental overview, J. Atmos. Terr. Phys., 55, 577, 1993.

Rishbeth, H. and Williams, P. J. S.: The EISCAT ionospheric radar: the system and its early results, Q. J. R. Astron. Soc., 26, 478, 1985.

Robinson, T. R.: The heating of the high-latitude ionosphere by high power radio waves, Phys. Rep., 179, 79-209, 1989.

Robinson, T. R., Stocker, A. J., Bond, G. E., Eglitis, P., Wright, D. M., and Jones, T. B.: O- and X-mode heating effects observed simultaneously with the CUTLASS and EISCAT radars and low power HF diagnostics at Troms $\emptyset$, Ann. Geophysicae, 15, 134, 1997.

Schulz, M. and Lanzerotti, L. J.: Particle diffusion in the radiation belts, Springer-Verlag, Berlin, Heidelberg, New York, 1974.

Shelley, E. G., Ghielmetti, A. G., Balsiger, H., et al.: The Toroidal Imaging Mass-Angle Spectrograph (TIMAS) for the Polar Mission, Space Sci. Rev., 71, 497, 1995.

Southwood, D. J.: A general approach to low-frequency instability in the ring current plasma, J. Geophys. Res., 81, 3340, 1976.

Southwood, D. J. and Kivelson, M. G.: Charged particle behaviour in low-frequency geomagnetic pulsations, 2. Graphical approach, J. Geophys. Res., 87, 1707, 1982.

Southwood, D. J., Dungey, J. W., and Etherington, R. J.: Bounce resonant interactions between pulsations and trapped particles, Planet. Space Sci., 17, 349, 1969.

Takahashi, K., Higbie, P. R., and Baker, D. N.: Characteristics of compressional Pc5 waves observed at geostationary orbit, J. Geophys. Res., 90, 1473, 1985.

Takahashi, K., McEntire, R. W., Lui, A. T. Y., and Potemra, T. A.: Ion flux oscillations associated with a radially polarised transverse Pc5 magnetic pulsation, J. Geophys. Res., 95, 3717, 1990.

Takahashi, K., Sato, N., Warnecke, J., Lühr, H., Spence, H. E., and Tonegawa, Y.: On the standing wave mode of giant pulsations, J. Geophys. Res., 97, 10717, 1992.

Walker, A. D. M., Greenwald, R. A., Stuart, W. F., and Green, C. A.: STARE auroral radar observations of Pc5 geomagnetic pulsations, J. Geophys. Res., 84, 3373, 1979.

Wilken, B., Weiß, W., Hall, D., Grande, M., Sørass, F., and Fennell, J. F.: Magnetospheric Ion Composition Spectrometer onboard the CRRES spacecraft, J. Spacecraft and Rockets, 29, 585, 1992.

Woch, J., Kremser, G., and Korth, A.: A comprehensive investigation of compressional ULF waves observed in the ring current, $\mathrm{J}$ Geophys. Res., 95, 15 113, 1990.

Wright, D. M. and Yeoman, T. K.: High resolution bistatic radar observations of ULF waves in artificially generated backscatter, Geophys. Res. Lett., 26, 2825-2828, 1999.

Wright, D. M., Yeoman, T. K., and Chapman, P. J.: High-latitude 
HF Doppler observations of ULF waves: 1. waves with large spatial scale sizes, Ann. Geophysicae, 15, 1548, 1997.

Wright D. M., Yeoman, T. K., Rae, I. J., Storey, J., Stockton-Chalk, A. B., Roeder, J. L., and Trattner, K. J.: Ground-based and Polar spacecraft observations of a giant $(P g)$ pulsation and its associated source mechanism, J. Geophys. Res., 106, 10 837, 2001.

Yeoman, T. K. and Wright, D. M.: ULF waves with drift resonance and drift-bounce resonance energy sources as observed in artificially-induced HF radar backscatter, Ann. Geophysicae, 19, 159, 2001.

Yeoman, T. K., Mao Tian, Lester, M., and Jones, T. B.: A study of Pc5 hydromagnetic waves with equatorward phase propagation, Planet. Space Sci., 40, 797, 1992.

Yeoman, T. K., Wright, D. M., Chapman, P. J., and Stockton-Chalk, A. B.: High-latitude observations of ULF waves with large azimuthal wave numbers, J. Geophys. Res., 105, 5453, 2000. 